\title{
Synthesis of isoprenoid diphosphate mimetics
}

\author{
Dimitar Vasilev, Ludger A. Wessjohann* \\ Leibniz Institute of Plant Biochemistry, Weinberg 3, 06120 Halle (Saale), Germany \\ *e-mail corresponding author:Wessjohann@ipb-halle.de
}

Keywords: isoprenoid diphosphate mimetics, inhibitors, prenyltransferases

\section{INTRODUCTION}

Cytokinins, are central regulators of cell division and differentiation in plants. ${ }^{1}$ Most naturally occurring cytokinins are 3,3-dimethylallyl adenine derivatives. Isopentenyladenine carries an unmodiefied isopentenyl side chain, whereas trans-zeatin and cis-zeatin carry hydroxylated side chains.

Isopentenyltransferases (IPTs) are known to be responsible for the biosynthesis of cytokinins. ${ }^{2}$ The model plant Arabidopsis thaliana contains nine isozymes (AtIPT1 to AtIPT9). One type of isopentenyl-transferases modifies tRNA and is called tRNA-isopentenyltransferases (AtIPT2 and AtIPT9). The other type (AtIPT1, AtIPT3-8) catalyzes the transfer of the isopentenyl moiety from dimethylallyl-diphosphate (DMAPP) to the $N^{6}$-amino group of an adenosine moiety (AMP, ADP or ATP) (Scheme 1).

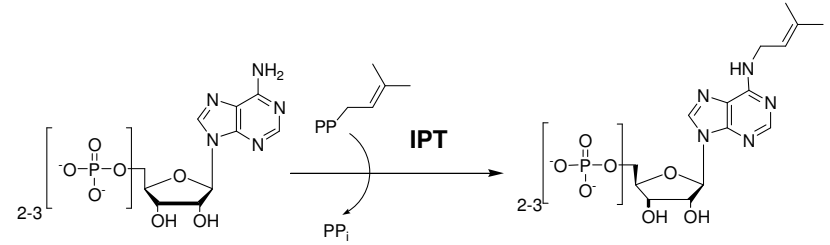

Scheme 1. Prenyl transfer reaction of adenosine phosphate (ADP or ATP) to isopentenyl adenosine phosphate catalyzed by AtIPT.

IPTs appear to be important targets in agricultural science. For this reason, their inhibition by synthetic substrates may prove useful in crop production.

\section{RESULTS AND DISCUSSION}

We focused our interest on the design and the synthesis of pyrophosphate mimics ${ }^{3}$ as competitive IPT inhibitors. Naturally occurring isoprenoid pyrophosphate substrates (e.g. DMAPP, geranylPP, farnesyl-PP) suffer enzymatic hydrolysis (Scheme 2).

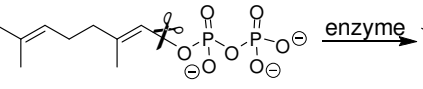

$$
\begin{aligned}
& \text { Geranyl-PP } \\
& \text { allylic cation }
\end{aligned}
$$

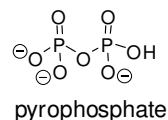

Scheme 2. Enzymatic hydrolysis of Geranyl-PP.
However, replacement of the biologically labile pyrophosphate group by bioisosteric moieties results in dephosphorylation-resistent analogues (Fig. 1). ${ }^{3-6}$

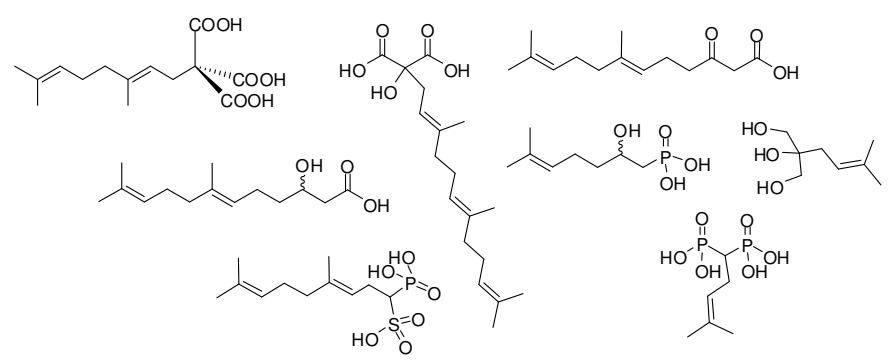

Figure 1: Examples of isoprenoid diphosphate surrogates.

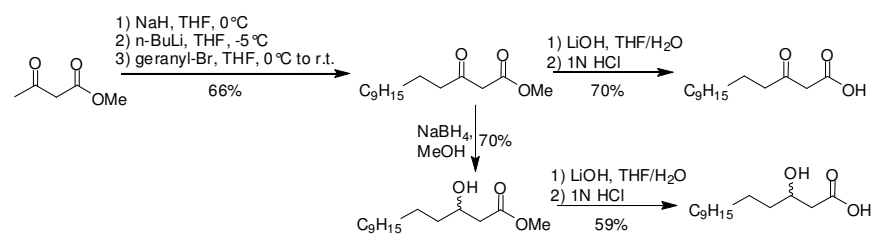

Scheme 3. Exemplary synthesis of analogues.

\section{CONCLUSION}

A series of several isoprenoid-derived pyrophosphate analogues have been synthesized. Biochemical studies on their activity as inhibitors of prenyltransferases are underway.

\section{ACKNOWLEDGEMENTS}

D. Vasilev thanks the Leibniz Institute of Plant Biochemistry for financial support.

\section{REFERENCES}

1 Mok, D. W.S.; Mok, M.C. Annu. Rev. Plant Physiol. Plant Mol.

Biol. 2001, 52, 89-118

2 Chu, H.-M.; Ko, T.-P.; Wang, A. H.-J. Nucleic Acids Research 2009, 37(1), 1-11

3 Wessjohann, L. A.; Fulhorst, M.; Zakharova, S.; Luczak, L. ARKIVOC 2004, xiii, 79.

4 Fairlamb, lan J.S; Dickinson, J. M.; O’Connor, R; Cohen, L. H.; van Thiel, C. F. Bioorganic Chemistry 2003, 31, 80-97

5 Wang, R.; Steensma, D. H.; Takaoka, Y.; Yun, W. J.; Kajimoto, T.; Wong, C.-H. Bioorganic \& Medicinal Chemistry, 1997, 5(4), 661-672

6 Magnin, D.R.; Biller, S.A.; Dickson, J.K. Jr; Logan, J.V.; Lawrence, R.M. Chen, Y. Sulsky, R.B. Ciosek, C.P. Jr; Harrity, T.W.; Jolibois, K.G.; et al. J. Med. Chem. 1995, 38, 2596-2605 